\title{
Metformin Versus Insulin for Pregnancy Outcomes in Gestational Diabetes: a Meta-Analysis
}

\author{
Fuchuan Wang*, Wei Yi, Jing Li, Li Li, Yuhong Hu, Dong Fu and Libin Sun ${ }^{1}$ \\ ${ }^{1}$ Department of Obstetrics and Gynecology, Beijing Di-Tan Hospital, Capital Medical University, China
}

*Corresponding author: Fuchuan Wang, Department of Obstetrics and Gynecology, Beijing Di-Tan Hospital, Capital Medical University, Beijing 100015, China.

To Cite This Article: Fuchuan Wang, Metformin Versus Insulin for Pregnancy Outcomes in Gestational Diabetes: a Meta-Analysis. 2020 - 10(1). AJBSR.MS.ID.001478. DOI: 10.34297/AJBSR.2020.10.001478.

Received: 眥 August 21, 2020; Published: 眥 August 26, 2020

\begin{abstract}
Background: The number of studies describing the use of metformin in gestational diabetes mellitus (GDM) is increasing, and metformin is considered an acceptable and economic alternative to insulin in many countries. Because metformin can pass through the placenta, it is necessary to continuously assess the maternal and fetal impact of metformin compared with insulin in pregnancy.

Methods and findings: We systematically searched PubMed, Embase, Medline, ClinicalTrials.gov, and the Cochrane database (from database inception to 10 February 2020) for randomized controlled trials (RCTs) that treatment with metformin versus insulin for GDM. Two reviewers independently assessed the articles, and conflicts were resolved by a third reviewer.

Main results: Twenty-seven RCTs ( $n=4568$ participants) were included for quantitative analyses in the meta-analysis. Metformin improved maternal outcomes, reduced the incidence of preeclampsia $(\mathrm{p}<0.00001$; risk ratio $(\mathrm{RR})=0.52$; confidence interval $(95 \% \mathrm{CI})[0.40,0.67])$, macrosomia $(\mathrm{p}<0.00001 ; \mathrm{RR}=0.62 ; 95 \% \mathrm{CI}[0.51,0.76])$. Metformin did not increase the incidence of premature delivery ( $\mathrm{p}=0.78 ; \mathrm{RR}=96 ; 95 \% \mathrm{CI}[0.61,1.45])$, small for gestational age (SGA) ( $\mathrm{p}=0.72$; $\mathrm{RR}=1.06$; $95 \% \mathrm{CI}[0.76,1.49])$. As for neonatal outcomes, metformin reduced the incidence of neonatal hypoglycemia ( $\mathrm{p}<0.00001 ; \mathrm{RR}=0.56 ; 95 \% \mathrm{CI}[0.48,0.64])$, neonatal intensive care unit admission ( $\mathrm{p}=0.001 ; \mathrm{RR}=0.78 ; 95 \% \mathrm{CI}[0.67,0.91])$.

Conclusions: According to our analysis of the literature, several adverse maternal and neonatal outcomes are reduced with the use of metformin. Metformin may have potential benefits for the mother and fetus. This meta-analysis protocol was registered with PROSPERO under registration number CRD42019148484. Keywords: metformin, insulin, gestational diabetes mellitus, meta-analysis, pregnancy outcome
\end{abstract}

\section{Introduction}

As a consequence of the obesity epidemic, the incidence of gestational diabetes mellitus (GDM) is rising and creating a heavy economic burden for individuals and the public health care system worldwide [1]. Unfortunately, GDM is related to significant, immediate and long-term adverse outcomes for both mother and child. Consequences of GDM include an increased risk of maternal cardiovascular disease, preeclampsia, cesarean delivery, type 2 diabetes and birth complications [2,3]. For the fetus, the key risks are accelerated intrauterine growth, resulting in macrosomia or large-for-gestational-age (LGA) neonates. This, in turn, can lead to shoulder dystocia, birth trauma, neonatal hypoglycemia, and admission to the neonatal intensive care unit (NICU) and a longer- term risk of obesity, type 2 diabetes, and cardiovascular disease in the child $[4,5]$. Consequently, it is essential to actualize effective clinical interventions to maintain glycemic control and limit fetal growth in GDM-affected pregnancies. Insulin resistance and beta-cell dysfunction are important mechanisms for the eventual development of GDM, and for this reason, insulin is an effective treatment for GDM [6.].

However, insulin treatment has several disadvantages: maternal weight gain, the need for multiple injections, higher cost and inconvenient modes of administration and monitoring $[8,9]$. These 2 disadvantages can decrease a patient's motivation to use insulin. Therefore, the identification of a safe, economic, convenient 
and effective method for glycemic control is particularly important in the management of GDM.

In contrast, metformin ( $\mathrm{N}, \mathrm{N}$-dimethylbiguanide), a biguanide oral glucose-lowering drug, can improve hepatic and peripheral sensitivity to insulin and is approved for use in the treatment of GDM in many countries across the world; additionally, metformin is a reasonable and safe first line pharmacological alternative to insulin for GDM in many countries [10,11]. However, metformin can cross the placenta and accumulate in fetal and placental tissues (at $50 \%-100 \%$ of the maternal concentration), and consequently, an increasing number of randomized clinical trials of metformin for GDM treatment have been carried out in many countries $[12,13]$. Insulin is still considered the first line of therapy for GDM by some professional organizations such as the American Diabetes Association. Therefore, it is very important to continuously evaluate the efficacy and safety of metformin treatment for pregnant women and their fetuses. The aim of our study was to determine whether metformin was superior to and safer than insulin for the treatment of pregnant women with GDM and their fetuses. This knowledge is particularly important because the number of pregnancies exposed to metformin have been increasing worldwide.

\section{Materials and Methods}

The three keywords and synonyms used to perform the literature search were metformin, gestational diabetes mellitus and randomized controlled trial. This meta-analysis was reported in accordance with the Preferred Reporting Items for Systematic Reviews and Meta-Analyses (PRISMA) guidelines. The systematic review protocol was registered in PROSPERO (CRD42019148484). Ethical approval was not required.

\section{Literature searches, search strategies, and eligibility criteria}

Three keywords and synonyms were used to perform systematic literature searches of PubMed, Embase, the Cochrane Central Register of Controlled Trials, the Cochrane library and Clinical Trials.gov. Neither filters nor language or location restrictions were applied to any of the searches. All references from the included trials and relevant previous systematic reviews were manually searched to avoid trials that might be missed in the electronic search. All randomized trials assessing women with GDM treated with metformin versus insulin therapy, published between 2003 and February 2020 and written in English were included. We accepted that GDM was diagnosed according to local diagnostic criteria. Trials were excluded as follows: (1) Studies comparing metformin to other oral hypoglycemic agents or interventions that were given prior to pregnancy; (2) Studies including participants with multiple pregnancies or preexisting diabetes; (3) Meeting abstracts and commentaries; or (4) Animal studies.

\section{Study selection, data extraction}

Each study was independently evaluated by two reviewers as per the inclusion/exclusion criteria. A third reviewer was available to resolve disputes where eligibility was unclear, and the team settled disagreements through discussion. The title and abstract were preliminarily selected, followed by a detailed screening of the full paper. The results of each step in the review process are recorded in the PRISMA flow diagram [Figure 1]. Two authors independently extracted data from eligible studies. The pregnancy outcome measures were weight gain after intervention, preeclampsia (BP of $140 / 90$ mmhg with proteinuria $>3 \mathrm{~g} / 24$ hours), prematurity (birth before 37 weeks), small for gestational age (SGA) (birth weight $<10$ th centile for gestational age), macrosomia (birth weight $>$ $4 \mathrm{~kg}$ ). Fetal outcome measures were NICU admission, neonatal hypoglycemia (two or more neonatal glucose values $<2.6 \mathrm{mmol}$ per liter) and neonatal respiratory distress.

\section{Quality assessment of included studies (risk of bias in individual studies)}

The quality and validity of each study was assessed independently by two authors using the Cochrane Collaboration tool for assessing risk of bias, which included seven categories: (1) Random sequence generation (selection bias); (2) Allocation concealment (selection bias); (3) Blinding of participants and personnel (performance bias); (4) Blinding of outcome assessment (detection bias); (5) Incomplete outcome data (attrition bias); (6) Selective reporting (reporting bias); and (7) Other bias. We classified these categories as low risk of bias, uncertain risk of bias or high risk of bias.

\section{Statistical Analysis}

The meta-analysis was performed using RevMan version $5.3[14]$. Heterogeneity was tested with the I 2 statistic (I $2 \geq 50 \%$ or $\mathrm{p}<0.1$ represented heterogeneity), and sensitivity analyses were performed using leave-one-out testing for individual studies. Metaanalyses use only the high-quality subset of studies. Funnel plots were constructed to assess publication bias. We calculated the mean difference (MD) for continuous data and the risk ratio (RR) for dichotomous data with 95\% confidence intervals (CIs). $\mathrm{P}<0.05$ indicated statistical significance.

\section{Results}

\section{Study selection}

Four hundred and fifty-two potentially relevant papers were retrieved by electronic searching of the specified databases, and 9 further studies were found via additional manual searching. A total of 237 records were retained after duplicates were removed. Then, after assessing the titles/abstracts, the investigators removed 47 systematic reviews and meta-analyses, 145 studies 
with inconsistent research content, 13 conference reviews and commentaries, and 1 animal study, and 31 trials received further consideration and full text assessment. After review of the full text, 4 studies were eliminated, and a total of 27 studies remained eligible for inclusion in this synthesis; all were suitable for the meta-analysis [15-41], representing 4,568 pregnancies. A total of 2280 patients were treated with metformin and 2288 patients were treated with insulin. [Figure 1] shows the process by which studies were selected. Seven standards were assessed in terms of risk of bias, and the risk of bias was low in the included studies; therefore, all studies were included. The risk-of-bias assessment is described in (Figure 2-3).

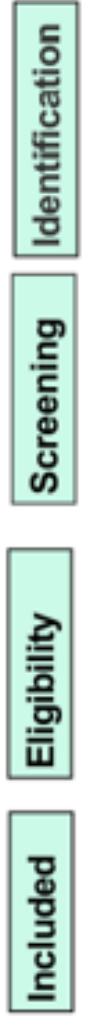

\section{Records identified by searching} in Pubmed, Embase databases $(n=323)$
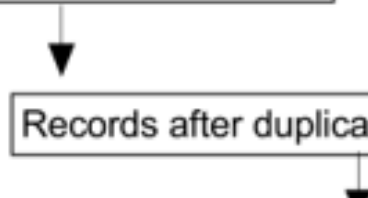
Title and abstract records screened $(n=237)$

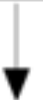

Studies selected for full text evaluation $(n=31)$

Records identified from other sources( $n=138)$,

Cochrane : $(n=129)$, Manual search $(n=9)$, Clinical Trials.gov: $(\mathrm{n}=0)$

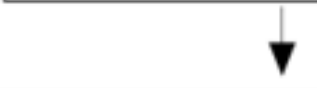

tes removed $(n=237)$

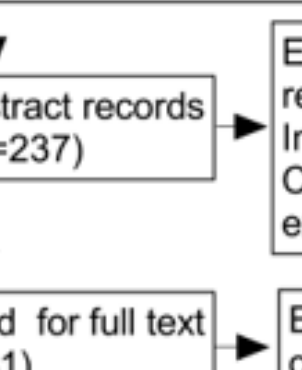

Excluded Comment $(n=4)$, Systematic review $(n=22)$, Meta-analysis $(n=25)$ Inconsistent research content( $n=145)$ Conference Review $(n=9)$,Animal experiment $(n=1)$

\section{Excluded Inconsistent research} content $(n=4)$

Studies included in the quantitative analysis (meta-analysis) $(n=27)$

\section{Random sequence generation (selection bias)}

\section{Allocation concealment (selection bias)}

Blinding of participants and personnel (performance bias)

Blinding of outcome assessment (detection bias)

Incomplete outcome data (attrition bias)

Selective reporting (reporting bias)

Other bias

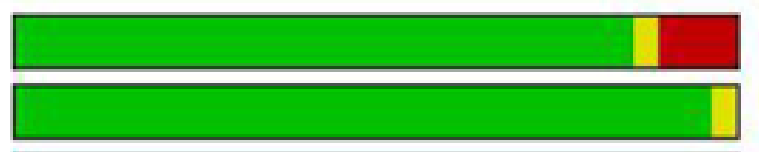

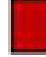

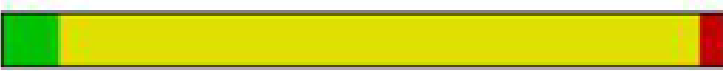

-
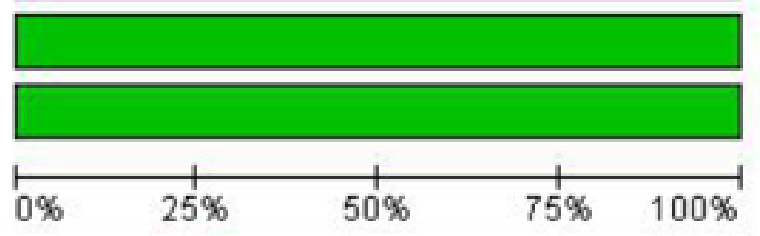


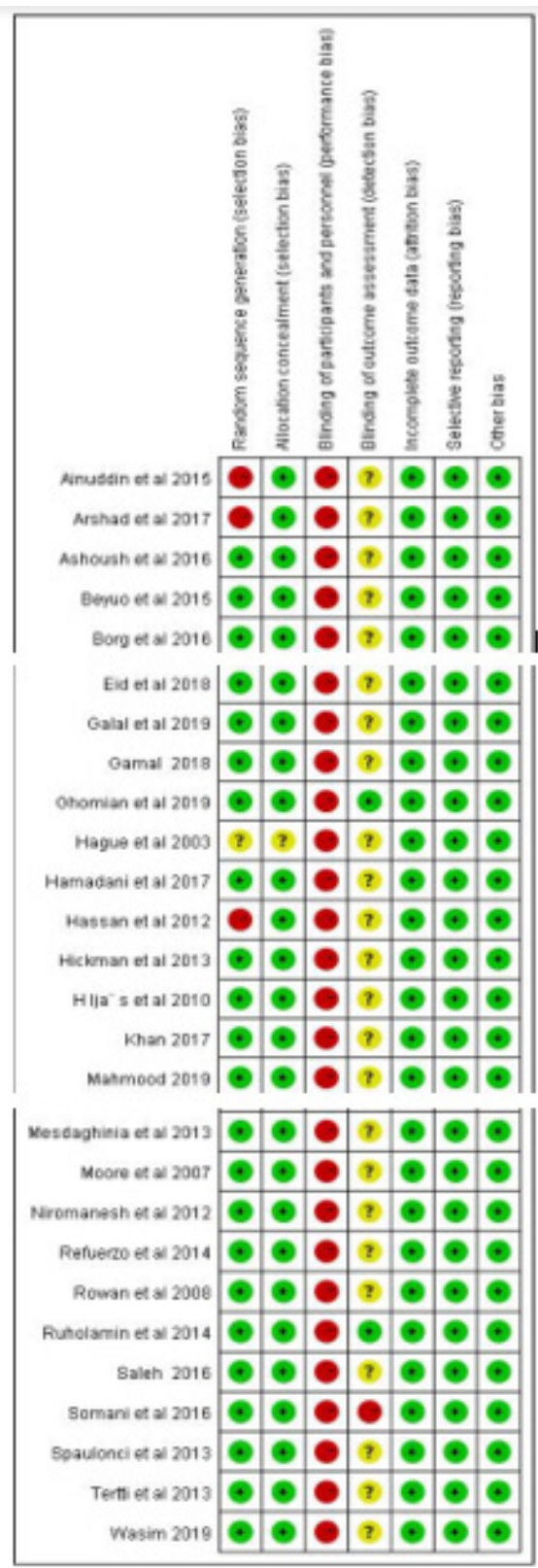

Figure 3: Risk-of-bias and methodological quality summary, containing each methodological quality item for each included study. +: low risk; "?”: unclear risk; -: high risk.

\section{Maternal outcomes}

There were no significant differences in maternal age, body mass index or gestational age before treatment in any of the included studies. Twelve studies ( $\mathrm{n}=2885$ patients) were included in the analysis of preeclampsia. Metformin reduced the risk of preeclampsia ( $\mathrm{p}<0.00001 ; \mathrm{RR}=0.52 ; 95 \% \mathrm{CI}[0.40,0.67]$; I 2=31\%). Six studies ( $n=2738$ patients) were included in the analysis of prematurity. Metformin can't increase the risk of preterm delivery $(\mathrm{p}=0.14 ; \mathrm{RR}=1.22 ; 95 \%$ CI $[0.94,1.58]$; I $2=3 \%)$. We compared the incidence of macrosomia in the two groups. Eighteen studies ( $n=2920$ patients) were included in the analysis of macrosomia, the results showed that macrosomia was lower by $38 \%$ in the metformin group than in the insulin group (RR 0.62, 95\% CI [0.51, 0.76 ], I $2=0 \%, p<0.00001$ ). Eight studies ( $n=1751$ patients) were analyzed the incidence of SGA. There was no difference in the incidence of SGA ( $p=0.72$; RR=1.06; 95\% CI [0.76, 1.49], I $2=0 \%$ ). Metformin reduces the incidence of macrosomia without increasing the risk of SGA infants (Figure 4).

\section{Neonatal outcomes}

Twenty studies ( $\mathrm{n}=3340$ patients) were included in the analysis of the incidence of neonatal hypoglycemia, and there was a difference between the two groups ( $\mathrm{p}<0.00001$; RR=0.56; 95\% CI [0.48, 0.64]; I $2=0 \%$ ), suggesting that metformin can significantly reduce the 
incidence of neonatal hypoglycemia compared with insulin. Fifteen studies also revealed that metformin can significantly reduce both the incidence of neonatal respiratory distress $(p=0.003 ; \mathrm{RR}=0.61$;
95\% CI $[0.44,0.85] ;$ I $2=12 \%)$ and the incidence of neonatal NICU admission ( $\mathrm{p}=0.001 ; \mathrm{RR}=0.78 ; 95 \%$ CI $[0.67,0.91]$; I $2=19 \%)$

(Figure 5).
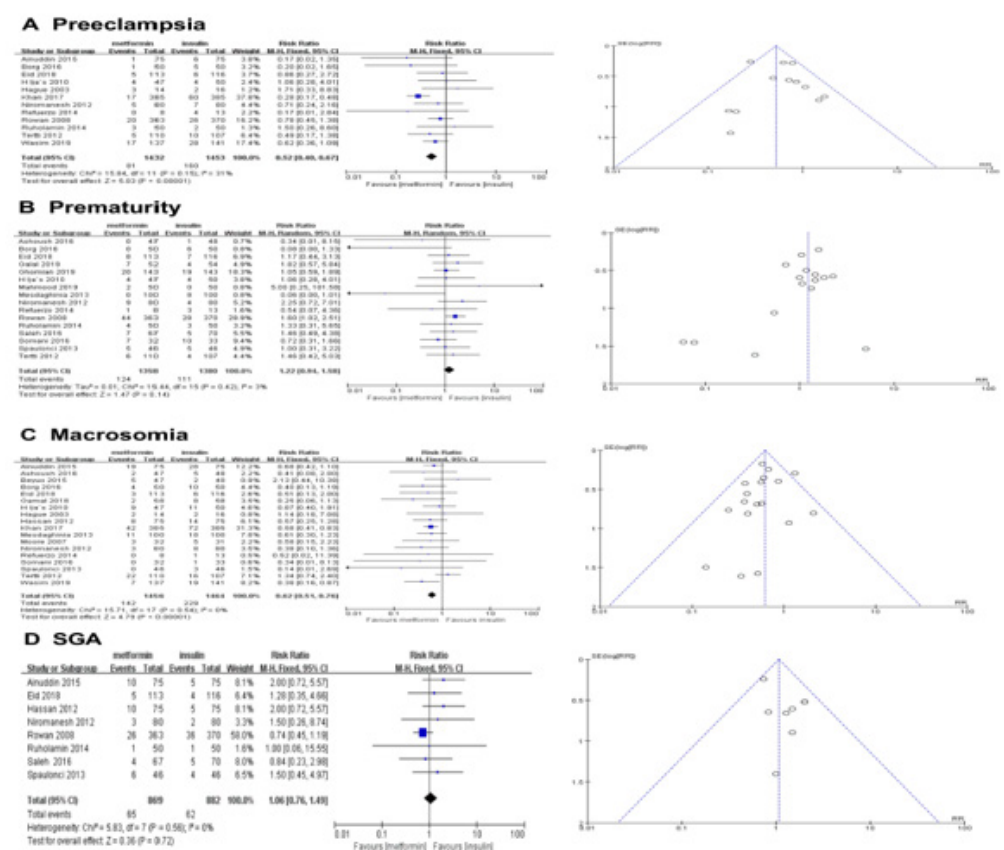

Figure 4: Forest plots and funnel plots for maternal outcomes. (A) Preeclampsia; (B) Premature delivery; (C) Macrosomia; (D) SGA. MD: Mean difference; Cl: Confidence interval; RR: Risk Ratio.

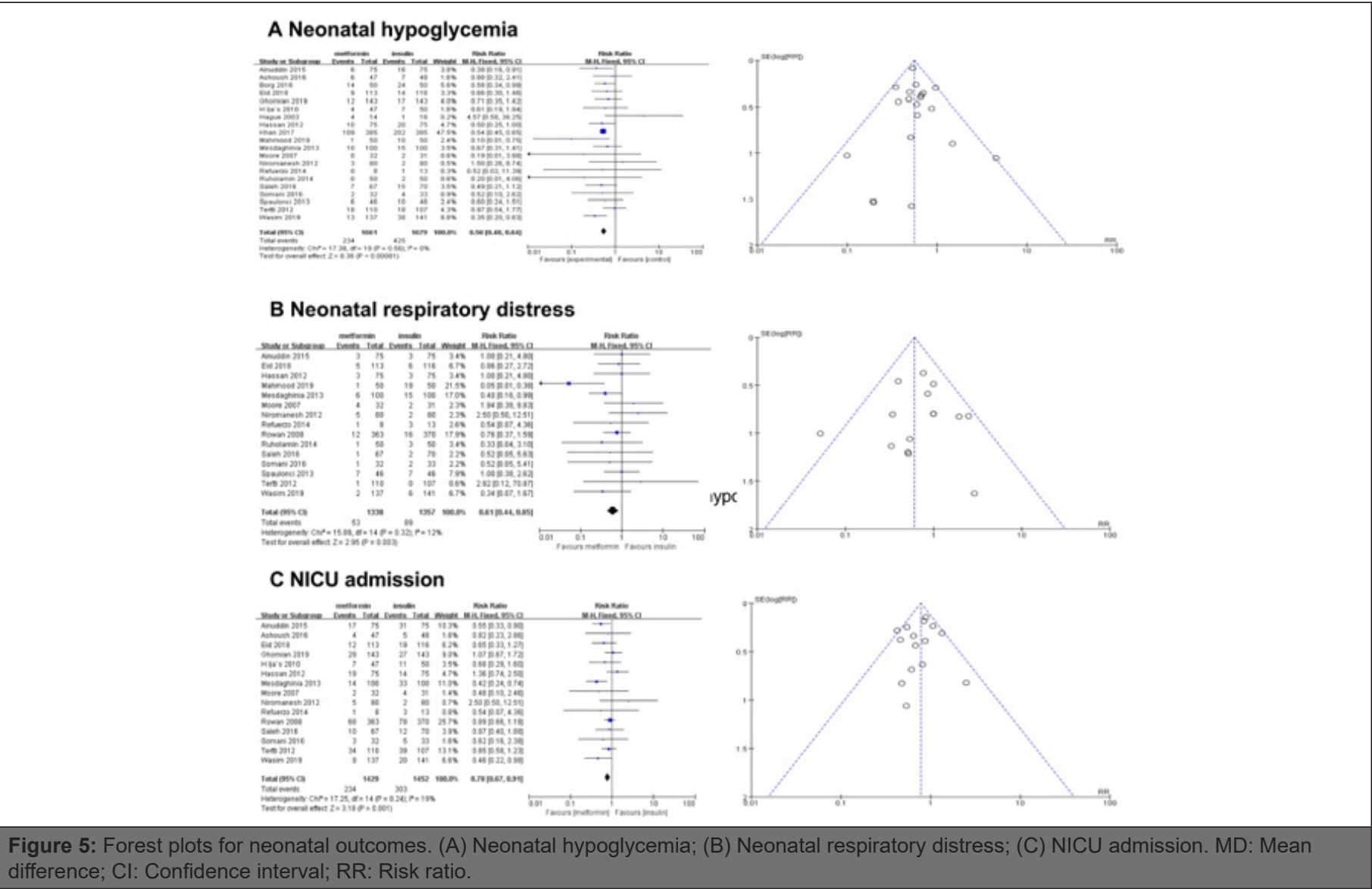




\section{Discussion}

Metformin can significantly improve the sensitivity of liver and peripheral tissue to insulin and reduce the output of liver glucose and the level of plasma glucose through direct and indirect effects on liver and muscle [42], and it is not associated with major complications or perinatal death[43], because of its advantages in terms of economy and convenience, metformin has been gradually used in the treatment of GDM. Although metformin can pass through the placenta, it has not been shown to be related to fetal malformations when used in the first three months of pregnancy [44]. To comprehend the safety and efficacy of metformin use in pregnancy, we searched several databases for reports of randomized controlled trials comparing insulin to metformin use in GDM, and identified twenty-seven randomized controlled trials, comprising 4568 patients, for inclusion in our meta-analysis.

Women with GDM have an increased risk of preeclampsia which may result in complications such as placental abruption, intrauterine growth retardation, premature birth and intrauterine death. Twelve studies ( $n=2885$ patients) were included in the analysis of preeclampsia. Our meta-analysis revealed that metformin can reduce the risk of preeclampsia, which is consistent with the findings of a previous review [45,4]. However, Le-xin Bao et al, who evaluated the incidence rate of preeclampsia by means of an analysis of nine studies involving 1813 GDM patients, noted that metformin did not reduce the risk of preeclampsia [46]. The reason for the disparity in our conclusions may be that we have included more studies in our analysis.

We analyzed sixteen studies in the incidence of preterm birth in both groups, and the results suggested that metformin does not increase the risk of preterm delivery, which is consistent with the results of a previous publication [46]. But several previous publications noted that metformin led to premature birth $[47,48]$. The difference of the above results may be due to the lack of studies included in the two analyses, Balsells M included 9 studies [47], Gui J included 5 studies [48]. These two analyses are earlier, with the gradual development of research, more and more research hints metformin does not increase the risk of preterm delivery in recent years.

We further analyzed the fetal growth, our results suggest that metformin can significantly reduce neonatal birth weight. In particular, metformin treatment reduces the incidence of macrosomia but does not concomitantly increase the risk of SGA, This is consistent with a study [49].

Our studies showed that metformin could significantly reduce the incidence rates of respiratory distress, NICU admission and neonatal hypoglycemia. Hypoglycemia is the most common complication of the newborn and the only readily preventable cause of neonatal brain injury [50], so metformin may reduce the risk of neonatal brain injury.

These findings support the notion that metformin perhaps is safe and beneficial in the treatment of GDM; however, information on long-term outcomes is still insufficient. Thus, more long-term follow-up studies are needed.

\section{Conclusion}

In summary, our results show that metformin can significantly reduce some complications of GDM among mothers and their infants compared with insulin. This provides support for the more conventional use of metformin as a treatment strategy that can improve the management of GDM.

\section{Acknowledgements}

This work is not supported by any organization.

\section{Statement of Ethics}

All analyses were based on the published studies, and thus, no patient consent was required.

\section{Disclosure statement}

No potential conflict of interest was reported by the authors.

\section{References}

1. Melchior H, KurchBek D, Mund D (2017) The prevalence of gestational diabetes. Dtsch Artztebl Int 114(14): 412-418.

2. Kampmann FB, Thuesen ACB, Hjort L, SjurdurFrodi Olsen, Sara Monteiro Pires, et al. (2019) Exposure to gestational diabetes is a stronger predictor of dysmetabolic traits in children than size at birth. J Clin Endocrinol Metab 104(5): 1766-1776.

3. Song C, Y Lyu, C Li, P Liu, J Li, et al. (2018) Long-term risk of diabetes in women at varying durations after gestational diabetes: A systematic review and meta-analysis with more than 2 million women. Obes Rev 19(3): 421-429.

4. Zhu Y, Zhang C (2016) Prevalence of gestational diabetes and risk of progression to type 2 diabetes: a global perspective. Curr Diab Rep 16(1): 7.

5. Chiefari E, Arcidiacono B, FotiD, A Brunetti (2017) Gestational diabetes mellitus: an updated overview. J Endocrinol Invest 40(9): 899-909.

6. Jasmine F Plows, Joanna L Stanley, Philip N Baker, Clare M Reynolds, Mark H Vickers, et al. (2018) The pathophysiology of gestational diabetes mellitus. Int J Mol Sci 19(11): 3342.

7. Riddle MC (2018) Introduction: standards of Medical Care in Diabetes-2018. Diabetes Care 41(Suppl 1): S1-S2.

8. Finneran MM, Landon MB (2018) Oral agents for the treatment of gestational diabetes. Curr Diabetes Rep 18(11): 119.

9. Moucheraud C, Lenz C, Latkovic M (2019) The costs of diabetes treatment in low-and middle income countries: a systematic review. BM] Glob Health 4(1): e001258.

10. World Health Organization. WHO model list of essential medicines: 20th list (March2017). Geneva: World Health Organization; 2017.

11. Society for Maternal-Fetal Medicine (SMFM) Publications Committee. SMFM Statement: pharmacological treatment of gestational diabetes. Am J Obstet Gynecol 218: B2-B4. 
12. Charles B, Norris R, Xiao X, William Hague (2006) Population pharmacokinetics of metformin in late pregnancy. Ther Drug Monit 28(1): 67-72

13. Sara Eyal, Thomas R. Easterling, Darcy Carr, Jason G Umans, Menachem Miodovnik, et al. (2010) Pharmacokinetics of metformin during pregnancy. Drug MetabDispos 38(5): 833-840.

14. R Core Team. R: a language and environment for statistical computing. Version 3.5.1. Vienna: R Foundation for Statistical Computing; 2018.

15. Ainuddin J, Karim N, Hasan AA, Sanower Ali Naqvi (2015) Metformin versus insulin treatment in gestational diabetes in pregnancy in a developing country: a randomized control trial. Diabetes Res Clin Pract 107(2): 290-299.

16. Arshad R, Khanam S, Shaikh F, Nasim Karim (2017) Feto-materna outcomes and Glycemic control in Metformin versus insulin treated Gestational Diabetics. Pak J Med Sci 33(5): 1182-1187.

17. Ashoush S, ElSaid M, Fathi H, Mohamed Abdelnaby (2016) Identification of metformin poor responders, requiring supplemental insulin, during randomization of metformin versus insulin for the control of gestational diabetes mellitus. J Obstet Gynaecol Res 42(6): 640-647.

18. Titus Beyuo, Samuel Amenyi Obed, Kenneth Kweku Adjepong-Yamoah, Kwasi Agyei Bugyei, Samuel Antwi Oppong, et al. (2015) Metformin versus Insulin in the Management of Pre-Gestational Diabetes Mellitus in Pregnancy and Gestational Diabetes Mellitus at the Korle Bu Teaching Hospital: A Randomized Clinical Trial. PLoS ONE 10(5): e0125712.

19. Borg HS, Ezat S (2016) Metformin opposed to insulin in the management of gestational diabetes. Res Obstet Gynecol 4: 17-26

20. Eid SR, Moustafa RSI, Salah MM (2018) Is metformin a viable alternative to insulin in the treatment of gestational diabetes mellitus (GDM)? Comparison of maternal and neonatal outcomes. Egypt Pediatr Assoc Gaz 66(1): 15-21.

21. Mohammad Galal, Walaa Mohammad El Bassioune (2019) Metformin versus insulin in treatment of gestational diabetes mellitus: A randomized controlled trial. Res J ObstetGynecol 12(1): 23-27.

22. Hatem El Gamal, Mohamed Abd Elaleem, SherifSadek, et al. (2018) Insulin versus Metformin in Treatment of Gestational Diabetes Mellitus (Randomized Controlled Clinical Trial).The Egyptian Journal of Hospital Medicine 72 (1): 3753-3761.

23. Nayereh Ghomian, Seyede Houra Mousavi Vahed, Samaneh Firouz Mohammad Ali Yaghoubi, Masoud Mohebbi, et al. (2019) The efficacy of metformin compared with insulin in regulating blood glucose levels during gestational diabetes mellitus: a randomized clinical trial. J Cell Physiol 234(4): 4695-4701.

24. Hague WM, Davoren PM, Oliver J (2003) Contraindications to use of metformin. Metformin may be useful in gestational diabetes. BMJ 326(7392): 762.

25. Hamadani A, Zahid S, Butt ZB (2017) Metformin versus insulin treatment in gestational diabetes in pregnancy and their effects on neonatal birthweight. Pak J Med Health Sci 11(3): 914-916.

26. Hassan JA, Karim N, Sheikh Z (2012) Metformin prevents macrosomia and neonatal morbidity in gestational diabetes. Pak J Med Sci 28(3): 384-389.

27. M Ashley Hickman, Ryan McBride, Kim A Boggess, Robert Strauss (2013) Metformin Compared with Insulin in the Treatment of Pregnant Women with Overt Diabetes: A Randomized Controlled Trial. Am J Perinatol 30(6):483-490.

28. Hljäs, M Vääräsmäki, L Morin-Papunen, R Keravuo, T Ebeling, et al. (2011) Metformin should be considered in the treatment of gestationa diabetes: a prospective randomised study. BJoG 118(7): 880-885.
29. Khan RMA, Mukhtar A, Khawar A (2017) Comparison of metformin with insulin in the management of gestational diabetes. Med Forum Mon 28: 105-109.

30. Mahmood OA (2019) Metformin versus insulin in the management of gestational diabetes mellitus. Med J Babylon 16(4): 346-350.

31. Elahe Mesdaghinia, Mansoureh Samimi, Zhila Homaei, Farzaneh Saberi, Seyyed Gholam Abbas Moosavi, et al. (2013) Comparison of newborn outcomes in women with gestational diabetes mellitus treated with metformin or insulin: a randomised blinded trial. Int J Prev Med 4(3): 327-333.

32. Lisa E Moore, Christian M Briery, Diana Clokey, Rick W Martin, Nancy J Williford, et al. (2007) Metformin and insulin in the management of gestational diabetes mellitus: preliminary results of a comparison. J Reprod Med 52(11): 1011-1015.

33. Shirin Niromanesh, Azin Alavi, Fatemeh Rahimi Sharbaf, Nooshin Amjadi, et al. (2012) Metformin compared with insulin in the management of gestational diabetes mellitus: a randomized clinical trial. Diabetes Res Clin Pract 98(3): 422-429.

34. Janet A Rowan, William M Hague, Wanzhen Gao, Malcolm R Battin, M Peter Moore, et al. (2008) Metformin versus insulin for the treatment of gestational diabetes. N Engl J Med 358(19): 2003-2015.

35. Ruholamin S, Eshaghian S, Allame Z (2014) Neonatal outcomes in women with gestational diabetes mellitus treated with metformin in compare with insulin: a randomized clinical trial. J Res Med Sci 19(10): 970-975.

36. Saleh HS, Abdelsalam WA, Mowafy HE (2016) Could metformin manage gestational diabetes mellitus instead of insulin? Int J Reprod Med 2016: 3480629.

37. Somani PS, Sahana PK, Chaudhuri P (2016) Treatment of gestational diabetes mellitus: insulin or metformin? J Evol Med Dent Sci 5(63): 4423-4429.

38. Cristiane PavaoSpaulonci, Lisandra Stein Bernardes, ThatianneCoutheux Trindade, Marcelo Zugaib, Rossana Pulcineli Vieira Francisco (2013) Randomized trial of metformin vs insulin in the management of gestational diabetes. Am J Obstet Gynecol 209(1): 34.e1-34.e7.

39. K Tertti, U Ekblad, P Koskinen, T Vahlberg, T Rönnemaa (2013) Metformin vs. insulin in gestational diabetes. A randomized study characterizing metformin patients needing additional insulin. Diabetes ObesMetab 15(3): 246-251.

40. TAYYIBA WASIM, SHYSTA SHAUKAT, LUBNA JAVAID, SAIMA MUKHTAR, WASIM AMER, et al. (2019) Comparison of Metformin and Insulin for Management of Gestational Diabetes Mellitus. PJMHS 13(3): 823-827.

41. Jerrie S Refuerzo, Rose Gowen, Claudia Pedroza, Maria Hutchinson, Sean C Blackwell,et al. (2015) A Pilot Randomized, Controlled Trial of Metformin versus Insulin in Women with Type 2 Diabetes Mellitus during Pregnancy. Am J Perinatol 30(2): 163-170.

42.E Cosson, L Carbillon, P Valensi (2017) High fasting plasma glucose during early pregnancy: A review about early gestational diabetes mellitus. J. Diabetes Res 2017: 8921712.

43. Priya G, S Kalra (2018) Metformin in the management of diabetes during pregnancy and lactation. Drugs Context 7: 212523.

44. Jorieke E H Bergman,L Renee Lutke, Rijk O B Gans,MarieClaude Addor, Ingeborg Barisic,et al. (2018) Beta-blocker use in pregnancy and risk of specific congenital anomalies: a European case-malformed control study. Drug Saf 41(4): 415-427.

45. S Butalia, L Gutierrez, A Lodha, E Aitken, A Zakariasen, et al. (2017) Shor and longterm outcomes of metformin comparedwith insulin alone in pregnancy: a systematic review and meta-analysis. Diabet Med34(1):2736. 
46. Lexin Bao, Wanting Shi, Yuxin Han (2019) Metformin versus insulin for gestational diabetes: a systematic review and meta-analysis, The Journal of Maternal-Fetal \&Neonatal Medicine.

47. BalsellsMontserrant, GarciaPatterson Apolonia, Sola Ivan, Roque Marta,GichIgnasi,et al. (2015) Glibenclamide, metformin, and insulin for the treatment of gestational diabetes: a systematic review and metaanalysis. BMJ 70(5): 305-307.

48. Gui J, Liu Q, Feng L (2013) Metformin vs insulin in the management of gestational diabetes: a meta-analysis. PLoS One 8(5): e64585.
49. Tarry Adkins JL, Aiken CE, Ozanne SE (2019) Neonatal, infant, and childhood growth following metformin versus insulin treatment for gestational diabetes: Asystematic review and meta-analysis. PLoSMed 16(8): e1002848.

50. Jane E Harding, Deborah L Harris, Joanne E Hegarty, Jane M Alsweiler Christopher Jd McKinlay, et al. (2017) An emerging evidence base for themanagement of neonatal hypoglycaemia. Early Hum Dev 104: 51-56. 